\title{
Probing Ferroelectric Polymers at the Nanoscale
}

\author{
Stephen Ducharme*
}

* Department of Physics and Astronomy, Nebraska Center for Materials and Nanoscience, University of Nebraska, Lincoln, NE 68588-0111

The properties of ferroelectric thin films and nanostructures are receiving increasing attention due in part to advances in nanoscale fabrication and characterization and in part due to the enormous commercial potential for application of highly integrated ferroelectric devices in a wide range of technologies, such as, nonvolatile memories, micro- and nano-electromechanical systems, ultrasonic transducer arrays, and infrared imaging. The purpose of this presentation is to review some of the more remarkable, and potentially useful, discoveries that have come out of experimental and theoretical advances in the investigation of ferroelectric polymers and oligomers based on vinylidene fluoride (VDF), or -(CH2-CF2)-. These are true (not liquid) crystalline systems and proper ferroelectrics, where polarization is the primary order parameter. The thin films and nanostructures are made by Langmuir-Blodgett deposition [1], which afforded a number of remarkable discoveries, such as the two-dimensional ferroelectricity, and intrinsic polarization switching[2]. They have also advanced the development of low-voltage nonvolatile memories and related technologies [3].

The VDF-based polymers can be structured into films as thin as one nanometer and nanocrystals as small as $5 \mathrm{~nm}$ thick by $90 \mathrm{~nm}$ in diameter (Fig. 1) [4], while maintaining their essential ferroelectric properties -- bistable polarization, piezoelectric response, and pyroelectric response. Piezoresponse force microscopy, for example, has been used to image polarization with a resolution of $6 \mathrm{~nm}$ and to write arbitrary polarization patterns with a resolution of $50 \mathrm{~nm}$ (Fig. 2) [5-7]. I will review recent results from nanoscale structural and functional studies of ferroelectric polymers, with an emphasis on the measurement and control of polarization at the nanoscale.

\section{References}

[1] A. V. Bune, V. M. Fridkin, S. Ducharme, L. M. Blinov, S. P. Palto, A. V. Sorokin, S. G. Yudin, and A. Zlatkin, "Two-Dimensional Ferroelectric Films," Nature (London), vol. 391, pp. 874-877, 26 Feb. 1998.

[2] S. Ducharme, S. P. Palto, and V. M. Fridkin, "Ferroelectric Polymer Langmuir-Blodgett Films," in Ferroelectric and Dielectric Thin Films. vol. 3, H. S. Nalwa, Ed. San Diego: Academic Press, 2002, pp. 545-591.

[3] S. Ducharme, T. J. Reece, C. M. Othon, and R. K. Rannow, "Ferroelectric Polymer Langmuir-Blodgett Films for Nonvolatile Memory Applications," IEEE Trans. Device Mater. Rel., vol. 5, pp. 720-35, December 2005.

[4] M. Bai and S. Ducharme, "Ferroelectric Nanomesa Formation from Polymer LangmuirBlodgett Films," Appl. Phys. Lett., vol. 85, pp. 3528-30, 2004.

[5] B. J. Rodriguez, S. Jesse, S. V. Kalinin, J. Kim, and S. Ducharme, "Nanoscale polarization manipulation and imaging of ferroelectric Langmuir-Blodgett polymer films," Appl. Phys. Lett., vol. 90, p. 122904, 2007.

[6] J. Kim, "Polarization Coupling in Nanostructured Ferroelectric Polymer Langmuir-Blodgett Films," in Department of Physics and Astronomy. vol. PhD Lincoln: University of Nebraska, 2008 , p. in preparation. 
[7] P. Sharma, T. J. Reece, D. Wu, V. M. Fridkin, S. Ducharme, and A. Gruverman, "Nanoscale Domain Patterns in Ultrathin Polymer Ferroelectric Films " J. Phys.: Condens. Matter, vol. 21, p. 485902, 2009.

[8] This research was supported by the National Science Foundation, the Office of Naval Research, the Department of Energy, and the Nebraska Research Initiative.
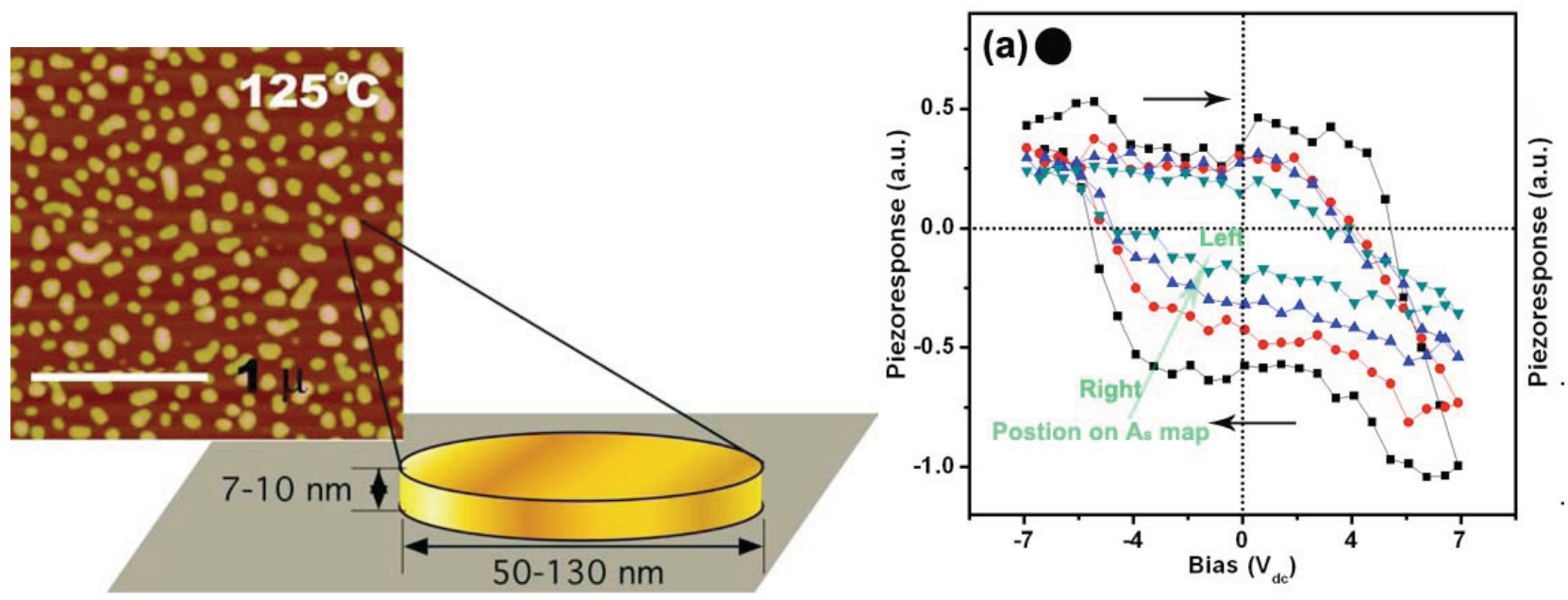

FIG. 1. Left: Atomic Force Microscopy (AFM) image of an array ferroelectric polymer nanomesas formed by self-assembly on a silicon wafer [4]. Each nanomesa is ferroelectric and supports multiple domains. Right: Polarization hysteresis loops from a ferroelectric polymer nanomesa recorded using Piezoresponse Force Microscopy (PFM) [6].
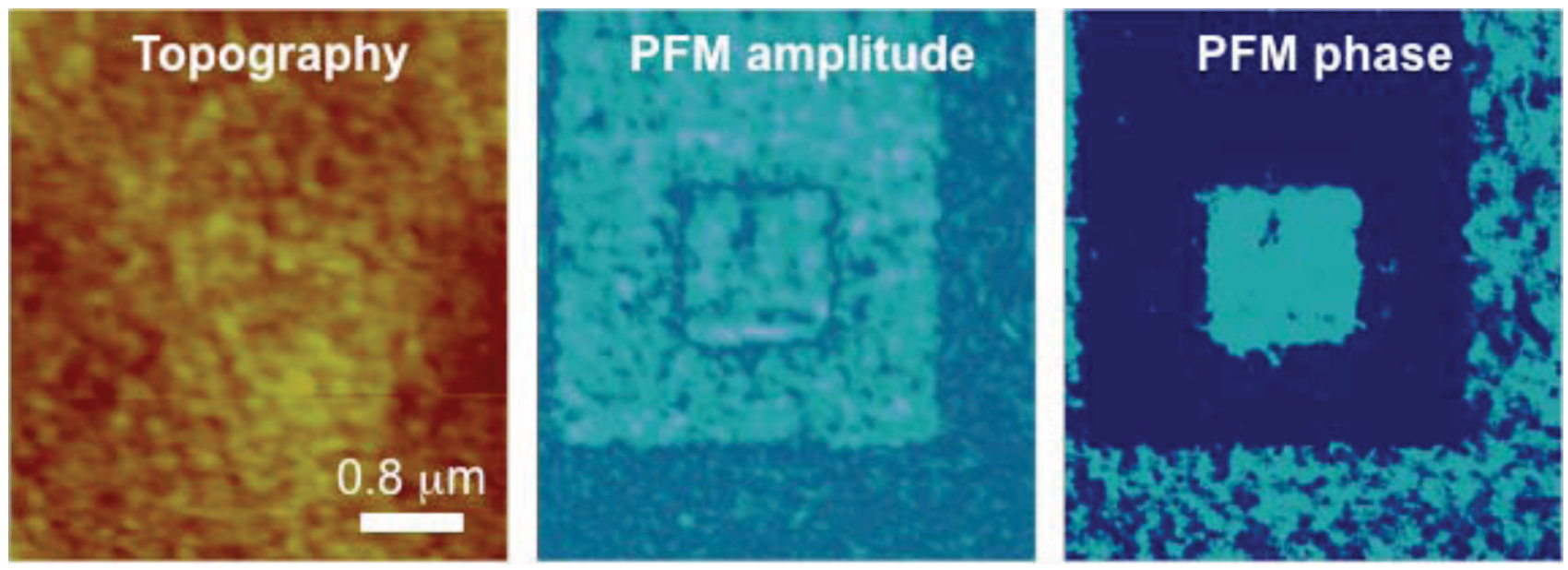

FIG. 2. Domain patterning in a ferroelectric polymer Langmuir-Blodgett film. Left: AFM topography shows a smooth film. Middle: PFM amplitude shows strong polarization signal over an area of approximately $2 \mu \mathrm{m}$ by $2 \mu \mathrm{m}$. Right: PFM phase image shows that the inner and outer squares were polarized in opposite directions by the PFM tip [5]. 